\title{
Preparation, characterization and in vitro evaluation of theophylline loaded microemulsion formulations
}

\author{
Tilbe Çevikelli ${ }^{1}$, Deniz Onan² ${ }^{2}$ Umay Merve Güven ${ }^{1}$, Esra Demirtürk ${ }^{1 *}$ \\ ${ }^{1}$ Department of Pharmaceutical Technology, Faculty of Pharmacy, Çukurova University, Adana, Turkey \\ ${ }^{2}$ Department of Pharmaceutical Biotechnology, Faculty of Pharmacy, Çukurova University, Adana, Turkey
}

\begin{tabular}{|c|c|}
\hline \multicolumn{2}{|c|}{ A RT ICLE I N F C } \\
\hline \multicolumn{2}{|c|}{ Article history: } \\
\hline Received & 08 Мау 2020 \\
\hline Revised & 20 May 2020 \\
\hline Accepted & 28 May 2020 \\
\hline Online & 01 Jun 2020 \\
\hline Published & 15 Jun 2020 \\
\hline \multicolumn{2}{|l|}{ Keywords: } \\
\hline \multicolumn{2}{|c|}{ Asthma therapy } \\
\hline \multicolumn{2}{|c|}{ Controlled release } \\
\hline \multicolumn{2}{|c|}{ Drug delivery system } \\
\hline \multicolumn{2}{|c|}{ Microemulsion } \\
\hline \multicolumn{2}{|c|}{ Theophylline } \\
\hline \multicolumn{2}{|c|}{$\begin{array}{l}\text { "Corresponding author: } \\
\text { demirturkesra@hotmail.com }\end{array}$} \\
\hline
\end{tabular}

\begin{abstract}
A B S T R A C T
Theophylline is widely used as a bronchodilator drug in asthma and chronic obstructive lung disease because it is effective and inexpensive. Theophylline blood concentrations should be monitored since its therapeutic index is narrow, its optimum blood levels range from 10 to 20 $\mu \mathrm{g} / \mathrm{mL}$; its serious side effects take place over $20 \mu \mathrm{g} / \mathrm{mL}$; its efficacy falls below $10 \mu \mathrm{g} / \mathrm{mL}$ blood concentrations. The aim of the present study is to formulate and characterize oil-in-water microemulsion systems for oral delivery of theophylline. Microemulsion formulations represent an interesting delivery vehicle for poorly water-soluble drugs, allowing for improving their solubility and dissolution properties. A simple and reliable UV-Spectrophotometric method was developed and validated for the determination of theophylline in the concentration range of 25 $150 \mu \mathrm{g} / \mathrm{mL}$. In vitro drug release profile showed that after 1 hour, $40 \%$ of the drug released through the formulation in Simulated Intestinal Fluid at $\mathrm{pH} 6.8$ as a release medium and the microemulsion formulations released more than $75 \%$ of the drug during the 5 hours study period. The globule size of drug-loaded formulations was in the range of $203.6 \pm 0.2 \mathrm{~nm}$. Results confirmed that the proposed microemulsion formulation containing theophylline could improve and control the drug release profile in comparison to the conventional dosage form.
\end{abstract}

This is an open-access article licensed under the Creative Commons Attribution 4.0 International License (CC-BY). (cc) BY

\section{INTRODUCTION}

Theophylline, which is a methylxanthine derivative, is widely used in the treatment of asthma because it is effective and cheap all over the world, including our country [1]. There is evidence of the anti-inflammatory and immunomodulating effects, as well as the bronchodilator effect [2]. When theophylline was first introduced into asthma therapy, it was used as a bronchodilator, and early dose-response studies showed an increasing acute bronchodilator response. Bronchial asthma is a chronic inflammatory disease of the airways. Bronchial asthma is a state of obstructive airway obstruction that is not related to any other disease, and hyperreactivity (hyperresponsiveness) of the airways against various stimuli [2,3]. Asthma is a serious social health problem for people of all age groups in the world. Asthma is a disease that significantly restricts the quality of life when it is uncontrolled, requires constant medical care and treatment, and can even lead to death [4]. Although it is claimed that there are approximately 300 million asthma patients worldwide today, the absence of a precise and generally accepted definition of asthma makes it difficult to make a reliable comparison of reported frequency values from various parts of the world $[5,6]$. Until recent years, asthma was considered a disease characterized by airway smooth muscle spasm, and bronchodilator drugs were used primarily in the treatment. Today, the main goal of asthma treatment is to suppress chronic inflammation in the airways, thereby controlling and maintaining the disease [2].

Drugs used in the treatment of asthma are divided into two as controlling and relaxing drugs. Controlling medicines are mainly used daily and long-term to keep asthma under control through its anti-inflammatory effects. Relaxing drugs, on the other hand, are medicines that act rapidly, correct bronchoconstriction, relieve symptoms, and are used when necessary [6]. Theophylline preparations especially provide controlled release, and they are included in the list of controlled medicines in the treatment of asthma.

The Global Initiative for Asthma Guidelines, 2002 guidelines for asthma, recommend that theophylline can be used as an add-on therapy to patients not controlled by low doses of inhaled corticosteroids but recommend long-acting $\beta_{2}$-agonists as more effective and with fewer adverse effects [7]. Several clinical studies have demonstrated that adding theophylline to inhaled corticosteroids in patients with mild to moderate asthma who are not controlled gives equivalent or better asthma control than doubling the dose of inhaled corticosteroids [8,9].

Theophylline was first used in the clinic in 1907 by Minkowski and a group of doctors as a diuretic in cardiac and renal edema following extraction from tea leaves by the 
German biologist Albrecht Kossel in 1888 [10]. It was added for the first time to The United States Pharmacopoeia's (USP) contents in 1918. With the development of pharmacokinetic science in the 1970s and 1980s, the pharmacokinetic properties of theophylline, the plasma level, and the relationship between bronchodilator effect and toxicity were intensively investigated. Considering this information, theophylline has become a commonly used drug in the treatment of asthma [11].

Microemulsions (MEs) are thermodynamically stable and translucent systems widely used for the systemic delivery of the drugs. In pharmaceutical fields, the interest in MEs is increasing; thus, they are applied to various administration routes. These systems form spontaneously at a certain concentration of oil, water, and surfactant. MEs often require the addition of cosurfactants to obtain an appropriate fluidity and flexibility of the interface. Due to their unique characteristics such as transparency, small droplet diameter (10-140 nm), high stability, and low surface tension, MEs are also one of the popular drug delivery systems for the controlled release of the active ingredient. Having very low inter-surface tension is essential in their formation and stability [12-14].

There are three types of MEs, including water-in-oil (w/ $\mathrm{o})$, oil-in-water $(\mathrm{o} / \mathrm{w})$, and bi-continuous systems. In w/o or $\mathrm{o} / \mathrm{w}$ type of MEs, the droplets are surrounded by a film made of surfactant and cosurfactant molecules. The main advantages of this type of system are the simple preparation method, low energy requirement, and also high encapsulation efficacy. The critical parameter for ME preparation is the correct selection of the oil phase and the surfactant/cosurfactant component in appropriate ratios.

Recently, there has been considerable interest in the ME formulations for the delivery of hydrophilic as well as lipophilic drugs as drug carriers because of its improved drug solubilization capacity, long shelf life, ease of preparation and improvement of bioavailability. The present study deals with the progress of polysorbate-based theophylline MEs in drug delivery.

\section{MATERIALS AND METHODS}

\subsection{Materials}

Theophylline powder, $\geq 99 \%$, anhydrous; isopropyl palmitate (IPP); Tween ${ }^{\circledR} 20$ and propylene glycol were provided from Sigma-Aldrich. All other chemicals were of analytical grade and used without further purification.

\subsection{Methods}

\subsubsection{Analytical method validation}

Method validation studies of theophylline were carried out according to the International Conference on Harmonization (ICH) guideline Q2(R1) [15]. The described method was validated with respect to linearity, precision, accuracy, the limit of detection (LOD) and quantitation (LOQ). The analysis method was developed by using a UVSpectrophotometer, and absorbance values were obtained at $295 \mathrm{~nm}$.

The stock solution of theophylline $(1 \mathrm{mg} / \mathrm{mL})$ was prepared by dissolving $50 \mathrm{mg}$ of drug in $50 \mathrm{~mL}$ of Simulated
Intestinal Fluid (SIF) (pH 6.8) prepared according to USP 29 [16]. The standard solutions were stored at $4 \pm 1^{\circ} \mathrm{C}$ in a clear glass volumetric flask and light protected with aluminum foil. Theophylline concentrations in the working solution chosen for the calibration curves were $25,50,75,100,125$, and $150 \mu \mathrm{g} / \mathrm{mL}$.

\subsubsection{Construction of pseudo-ternary phase diagrams}

Pseudo-ternary phase diagrams were obtained with a water titration method at room temperature $\left(25^{\circ} \mathrm{C}\right)$. For each phase diagram, the oil phase and the surfactant mixture $\left(\mathrm{S}_{\text {mix }}\right)$ were mixed and different ratios of oil:surfactant mixtures were prepared from 9:1, 8:2, 7:3, 6:4, 5:5, 4:6, 3:7, 2:8, and 1:9. Water was added drop by drop to each mixture carefully until the mixture became turbid at a certain point. The mixture that remained transparent and homogeneous after stirring was assigned to the monophasic area of the phase diagram. This process was repeated in the same way for each phase diagram $[17,18]$.

\subsubsection{Preparation of microemulsion formulation}

The pseudo-ternary phase diagram of the largest area was selected to determine the concentration range of the components in the ME. ME formulations for further studies were selected from the weight center of these pseudo-ternary diagrams [19].

\subsubsection{Globule size, zeta potential and polydispersity index}

The mean droplet size and size distribution (polydispersity index; PDI) of MEs were characterized by photon correlation spectroscopy (Nano ZS, Malvern, UK). Three individual runs were performed on each sample. The size of droplets is expressed as Z-average diameter and reported as the mean diameter. The surface charge of MEs was determined by measuring the zeta potential of MEs using the electrophoretic light-scattering technique (Nano ZS, Malvern, UK). For each sample, three individual runs were performed for which zeta potential was calculated as the mean value.

\subsubsection{Centrifugation}

ME formulation was centrifuged at $3000 \mathrm{rpm}$ for 30 minutes and then was examined for whether the system was monophasic or biphasic.

\subsubsection{In vitro drug release studies}

In vitro release tests were performed using dialysis membrane methods. The medium was $50 \mathrm{~mL}$ of Simulated Intestinal Fluid (SIF) pH 6.8 [16], and the temperature was kept at $37 \pm 0.5^{\circ} \mathrm{C}$. MEs containing the same amount of theophylline prepared based on the solubility study were directly introduced into the dissolution medium. While stirring the release medium using the magnetic stirrer at 100 $\mathrm{rpm}, 1 \mathrm{~mL}$ of dissolution medium was withdrawn at the predetermined period ( $5 \mathrm{~min}, 15 \mathrm{~min}, 30 \mathrm{~min}, 60 \mathrm{~min}, 90$ min, $2 \mathrm{~h}, 3 \mathrm{~h}, 4 \mathrm{~h}, 5 \mathrm{~h}$ ). After each sampling, $1 \mathrm{~mL}$ of fresh medium was added to the incubation medium. The amount of released active agent in the medium collected was determined by UV-spectroscopy, and cumulative release percentage of theophylline was calculated [20,21]. 


\section{RESULTS AND DISCUSSION}

\subsection{Analytical Method Validation}

The linearity between absorbance and concentration was analyzed using a calibration curve obtained from standard solutions of theophylline ( 25 to $150 \mu \mathrm{g} / \mathrm{mL}$ ). Linearity was evaluated by linear regression analysis, which was calculated by the least-square regression analysis. The determination coefficient $\left(\mathrm{R}^{2}\right)$ of 0.9991 was obtained. Linearity test results are shown in Table 1, and the regression curve is presented in Figure 1. In this study, the range was observed linearly to the highest concentration, and the $\mathrm{R}^{2}$ of 0.9991 was highly significant [18].

Table 1. Series and absorbance values of the linearity study

\begin{tabular}{cccccc}
\hline $\begin{array}{c}\text { Conc. } \\
(\boldsymbol{\mu g} / \mathbf{m L})\end{array}$ & SET 1 & SET 2 & SET 3 & Mean & SD \\
\hline 25 & 0.077 & 0.081 & 0.076 & 0.078 & 0.003 \\
50 & 0.172 & 0.154 & 0.158 & 0.161 & 0.009 \\
75 & 0.271 & 0.245 & 0.233 & 0.250 & 0.019 \\
100 & 0.333 & 0.320 & 0.295 & 0.316 & 0.019 \\
125 & 0.421 & 0.388 & 0.366 & 0.392 & 0.028 \\
150 & 0.513 & 0.485 & 0.429 & 0.475 & 0.043 \\
\hline
\end{tabular}

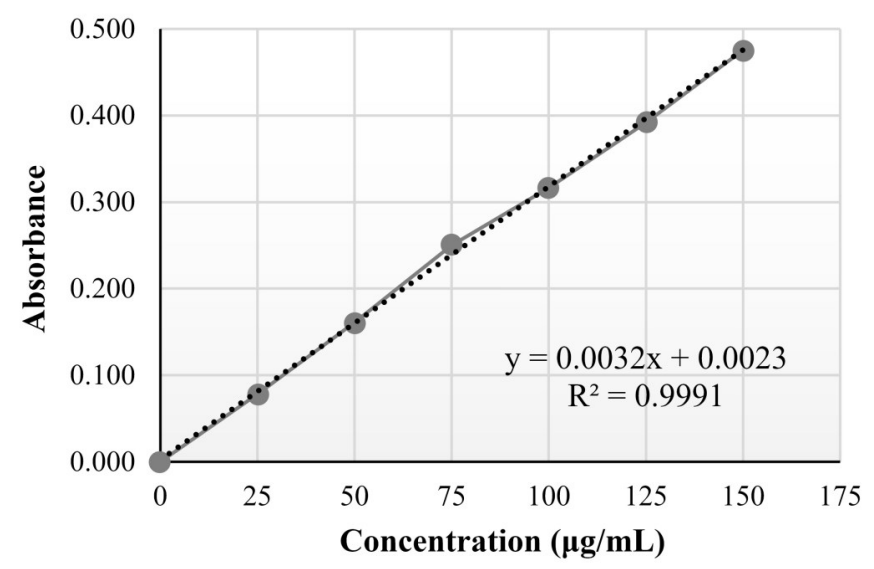

Figure 1. Regression profile of theophylline

$25,75,125 \mu \mathrm{g} / \mathrm{mL}$ theophylline solutions were analyzed three times in order to evaluate method precision, standard deviation (SD), and relative standard deviation (RSD). Results of precision and repeatability tests on different concentrations are given in Table 2.

Since the RSD\% values for both intermediate precision and repeatability were $<2 \%$, the method developed for theophylline was found to be precise according to the suggestions in ICH Q2(R1) guidelines [22].

The accuracy of an analytical method is the closeness of test results obtained by the method to the true value and is defined recovery. The prepared three standard solutions (50, $100,150 \mu \mathrm{g} / \mathrm{mL}$ ) were analyzed three times to evaluate the accuracy of the method developed. As given in Table 3, the recoveries of theophylline at various concentrations were obtained between $97-106 \%$. Since the percentage of recovery has been found between the acceptance criteria, which is $90-110 \%$, the analysis system for the determination of assay is verified. Low values of standard deviation denoted good repeatability of the measurement [23].
Table 2. Precision results of theophylline on three different days

\begin{tabular}{|c|c|c|c|c|c|c|}
\hline & \multicolumn{3}{|c|}{ Absorbance } & \multicolumn{3}{|c|}{ Concentration $(\mu \mathrm{g} / \mathrm{mL})$} \\
\hline & Day 1 & Day 2 & Day 3 & Day 1 & Day 2 & Day 3 \\
\hline \multirow{6}{*}{ 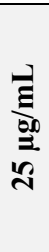 } & 0.076 & 0.107 & 0.076 & 23.031 & 32.719 & 23.031 \\
\hline & 0.078 & 0.106 & 0.078 & 23.656 & 32.406 & 23.656 \\
\hline & 0.078 & 0.108 & 0.077 & 23.656 & 33.031 & 23.344 \\
\hline & & & Mean & 23.448 & 32.719 & 23.344 \\
\hline & & & SD & 0.361 & 0.313 & 0.313 \\
\hline & & & RSD & 1.539 & 0.955 & 1.339 \\
\hline \multirow{6}{*}{ 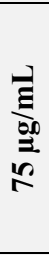 } & 0.274 & 0.25 & 0.241 & 84.906 & 77.406 & 74.594 \\
\hline & 0.271 & 0.245 & 0.230 & 83.969 & 75.844 & 71.156 \\
\hline & 0.269 & 0.240 & 0.229 & 83.344 & 74.281 & 70.844 \\
\hline & & & Mean & 84.073 & 75.844 & 72.198 \\
\hline & & & SD & 0.786 & 1.563 & 2.081 \\
\hline & & & RSD & 0.935 & 2.060 & 2.882 \\
\hline \multirow{6}{*}{ 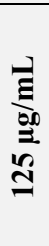 } & 0.419 & 0.389 & 0.361 & 130.219 & 120.844 & 112.094 \\
\hline & 0.420 & 0.386 & 0.366 & 130.531 & 119.906 & 113.656 \\
\hline & 0.425 & 0.390 & 0.370 & 132.094 & 121.156 & 114.906 \\
\hline & & & Mean & 130.948 & 120.635 & 113.552 \\
\hline & & & SD & 1.005 & 0.651 & 1.409 \\
\hline & & & RSD & 0.767 & 0.539 & 1.241 \\
\hline
\end{tabular}

Limit of detection (LOD) of an analytical procedure is the minimum amount of analyte which can be detected in a sample, while the limit of quantitation (LOQ) is the lowest concentration of an analyte in a test and can be determined with acceptable precision and accuracy under the specified test conditions. LOD and LOQ were determined using the calibration curve method, according to ICH Q2(R1) recommendations. The LOD and LOQ of the proposed method were calculated using the following equations:

$$
\begin{aligned}
& \mathrm{LOD}=3.3 \times \sigma / \mathrm{s} \\
& \mathrm{LOQ}=10 \times \sigma / \mathrm{s}
\end{aligned}
$$

where,

$\sigma=$ the standard deviation of the response

$\mathrm{s}=$ slope of the calibration curve [24].

LOD and LOQ for theophylline were found to be 0.260 $\mu \mathrm{g} / \mathrm{mL}$ and $0.786 \mu \mathrm{g} / \mathrm{mL}$, respectively.

\begin{tabular}{|c|c|c|c|}
\hline & Absorbance & Conc. $(\mu \mathrm{g} / \mathrm{mL})$ & Recovery \% \\
\hline \multirow{5}{*}{ 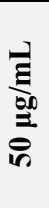 } & 0.157 & 48.344 & 96.688 \\
\hline & 0.154 & 47.406 & 94.813 \\
\hline & 0.163 & 50.219 & 100.438 \\
\hline & & Mean & 97.313 \\
\hline & & SE & 2.864 \\
\hline \multirow{5}{*}{ 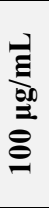 } & 0.333 & 103.344 & 103.344 \\
\hline & 0.337 & 104.594 & 104.594 \\
\hline & 0.334 & 103.656 & 103.656 \\
\hline & & Mean & 103.865 \\
\hline & & SE & 0.651 \\
\hline \multirow{5}{*}{ 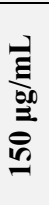 } & 0.512 & 159.281 & 106.188 \\
\hline & 0.514 & 159.906 & 106.604 \\
\hline & 0.512 & 159.281 & 106.188 \\
\hline & & Mean & 106.326 \\
\hline & & SE & 0.241 \\
\hline
\end{tabular}

Table 3. Accuracy results of theophylline

SE: Standard error 

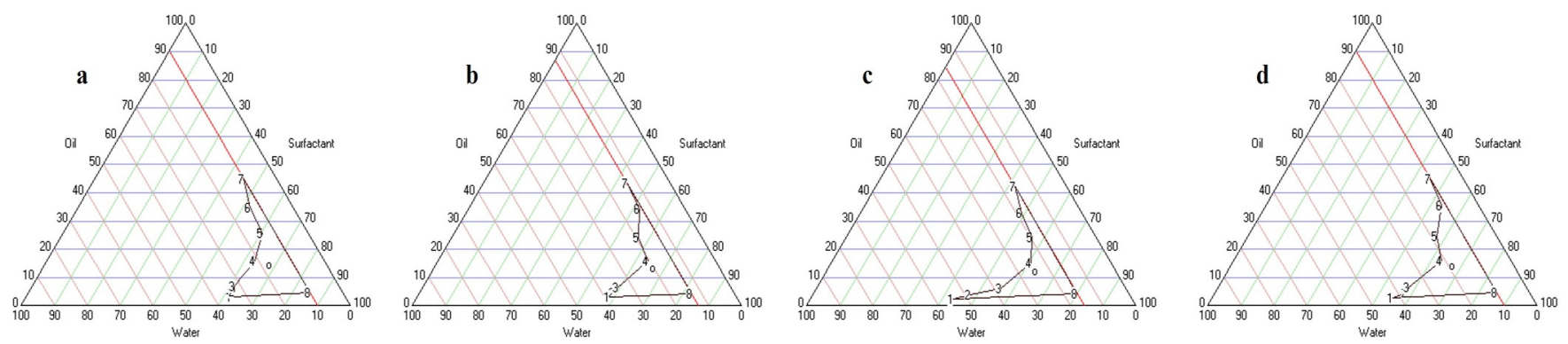

Figure 2. Pseudo-ternary phase diagrams of microemulsions surfactants:cosurfactant ratio a: 2:1, b: 3:1, c: 4:1, d: 5:1

\subsection{Construction of Pseudo-Ternary Phase Diagrams}

MEs are transparent, thermodynamically stable and isotropic oil, water, surfactant, and cosurfactant mixtures. Several studies have shown that ME formulations provide improved oral and dermal drug delivery properties [25,26]. Many ME formulations are based on short- and mediumchain alcohols, while others are based on poloxamer or propylene glycol as cosurfactants in combination with polysorbates [25].

It is known that large proportions of surfactant may cause irritation and toxicity. It is important to determine the surfactant concentration and to use the minimum concentration required for the development of the $\mathrm{ME}$ formulation [27].

In this study, all the ingredients were pharmaceutically acceptable and generally regarded as in the safe category. Nonionic surfactants are generally considered as having low toxicity and irritancy and, therefore, regarded to be suitable for oral administration.

Pseudo-ternary diagrams are equilateral triangles that define the compositional phase behavior of the system. The vertices represent pure ingredient or two ingredients, the edges indicate two-ingredient systems, and the interior represents all three ingredients in the system. Pseudo-ternary phase diagrams of the ME area of existence with isopropyl palmitate, Tween ${ }^{\circledR} 20$, propylene glycol, and water mixtures and the final compositions are presented in Figure 2. The ME formulations 5:1 ratio was selected, which showed larger ME regions. The complete composition of oil, surfactant, cosurfactant, and the aqueous phase were shown in Table 4.

Table 4. Composition of optimum microemulsion formulation

\begin{tabular}{lc}
\hline Components & $\mathbf{\%}$ \\
\hline Isopropyl palmitate & 14.5 \\
Tween ${ }^{\circledR} 20$ & 57.4 \\
Propylene glycol & 9.6 \\
Water & 18.6 \\
\hline
\end{tabular}

\subsection{Characterization of Microemulsion Formulation}

The final ME formulation maintained the predetermined characteristics after the addition of theophylline. Blank formulation and formulation including the active agent were clear transparent liquids with a homogenous appearance, no precipitation, good fluidity, and a yellow tinge at room temperature. When physical stability was observed, MEs showed no phase separation after centrifugation. Besides, there was no sign of turbidity or creaming.
The zeta-size analyzer was employed to determine the globule size of the ME formulation (Figure 3). The globule size was found to be $203.6 \pm 0.2 \mathrm{~nm}$. This shows that the optimized formulation was nano-sized and could be termed as nano formulations. Smaller emulsion globule size results in lower emulsification time and a larger surface area provided for the drug absorption. The small globule size and good dispersibility of the ME indicated that the bioavailability and absorption of theophylline could be improved. There was no significant change in globule size and PDI after active agent incorporation [28-30].

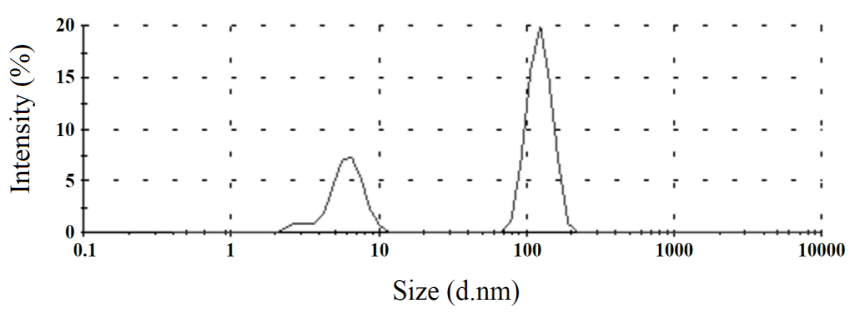

Figure 3. Globule size analysis of 5:1 microemulsion formulation

The globule size and polydispersity index have a significant impact on the physical stability of colloidal drug delivery systems when used to orally. The polydispersity index varies from 0.0 to 1.0 and is a measure of particle homogeneity. The globules are more homogeneous if the polydispersity index value is closer to zero [28,31]. The small polydispersity index of ME showed uniformity in the size distribution of globules, polydispersity index below 0.3 could be used as an indication of uniformity of globules. This result used as a determination of the stability of MEs [31].

The zeta potential is a method to measure the surface charge of particles or globules. Zeta potential is used for predicting dispersion stability, and its value depends on the physicochemical property of the drug, polymer, vehicle, presence of electrolytes, and their adsorption [29]. Figure 4 indicates the value of the zeta potential of the optimized ME. Zeta potential is an essential property of the formed dispersion, and large zeta potential value of nanostructures was an important factor for physical stability.

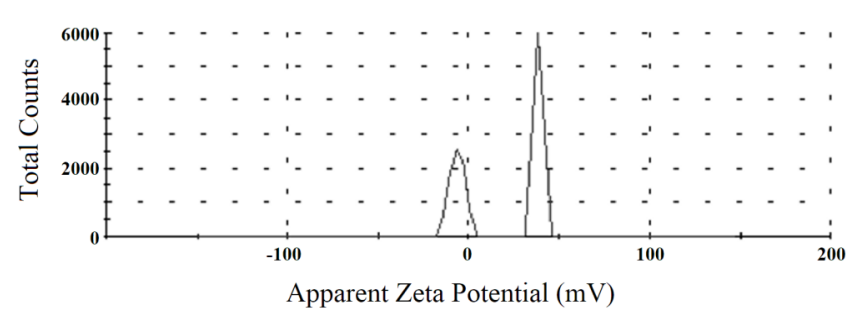

Figure 4. Zeta potential of 5:1 microemulsion formulation 
The values of zeta potential indicate that the system is relatively stable. The zeta potential values of $\mathrm{ME}$ may approach neutral charge due to the presence of the nonionic surfactant. The ME shows positive zeta potential, which is important for achieving the interaction of the gastrointestinal system for effective and prolonged theophylline delivery [30].

\subsection{In Vitro Drug Release Studies}

The dialysis bag is the most commonly used technique in the literature for drug release measurements [32]. The in vitro dissolution study of theophylline loaded formulation was compared with the dissolution profile of the pure theophylline. The cumulative release percentages at the regular time were calculated and showed in Figure 5. In the figure, the cumulative drug release percentage after 1 hour was $40 \%$ and $95 \%$ for ME formulation and pure active agent solution in SIF pH 6.8, respectively.

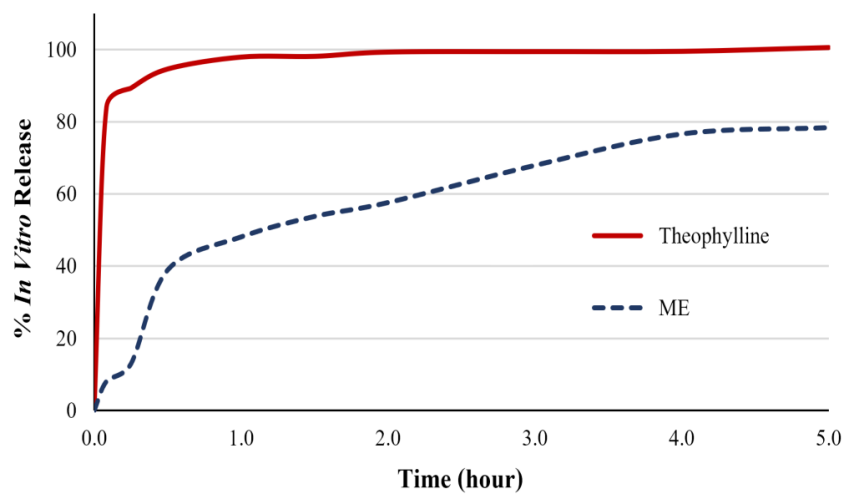

Figure 5. In vitro cumulative drug release profile

Experimental results showed that ME formulations release more than $75 \%$ of the drug during 5 hours of study period. These drug release behaviors confirm the sustained release of theophylline in the ME formulation, which enhances the drug retention time. The sustained release of this formulation is consistent with the literature [28-30].

\section{CONCLUSION}

In this study, we successfully prepared theophylline loaded ME formulation sized around $200 \mathrm{~nm}$. This study shows that the o/w ME with good clarity, ideal stability, and proper characterization, can be prepared successfully using Tween ${ }^{\circledR} 20$ as the surfactant and propylene glycol as the cosurfactant. Tween ${ }^{\circledR} 20$ :propylene glycol in the weight ratio of 5:1 was the surfactant providing greater area. The in vitro studies demonstrated the potential of developed ME for oral delivery of theophylline.

\section{AUTHOR CONTRIBUTIONS}

Concept: TÇ, DO, UMG, ED; Design: TÇ, DO, UMG, ED; Supervision: TÇ, DO, UMG, ED; Materials: TÇ, DO, UMG, ED; Data Collection and/or Processing: TÇ, DO, UMG, ED; Analysis and/or Interpretation: TC, DO, UMG, ED; Literature Search: TÇ, DO, UMG, ED; Writing: TÇ, DO, UMG, ED; Critical Reviews: TÇ, DO, UMG, ED.

\section{ACKNOWLEDGMENTS}

None.

\section{CONFLICT OF INTEREST DECLARATION}

The authors report no conflict of interest. The authors alone are responsible for the content and the writing of the paper.

\section{REFERENCES}

[1] Undem BJ, Lichtenstein LM. Drugs used in the treatment of asthma. In: Hardman JG, Limbird LE, editors. Goodman and Gilman's the Pharmacological Basis of Therapeutics. 10th ed. New York: McGraw -Hill; (2001). p. 733-754.

ISBN:9780071354691

[2] Kayaalp SO. Rasyonel Tedavi Yönünden Tıbbi Farmakoloji. 11th ed. Ankara: Hacettepe-Taş Kitapçllı; (2005). p. 594-611.

ISBN:9789759534158

[3] Kobzik L, Schoen FJ. The Lung. In: Cotran RS, Robbins SL, Kumar $\mathrm{V}$, editors. Robbins pathologic basis of disease. 5th ed. United States of America: W. B. Saunders Company; (1994). p. 673-734. ISBN:9780721650326

[4] D'Amato G, Vitale C, Molino A, Stanziola A, Sanduzzi A, Vatrella A, Mormile M, Lanza M, Calabrese G, Antonicelli L, D’Amato M. Asthma-related deaths. Multidiscip Respir Med. (2016);11(37):1-5. https://doi.org/10.1186/s40248-016-0073-0

[5] Bateman ED, Hurd SS, Barnes PJ, Bousquet J, Drazen JM, FitzGerald M, Gibson P, Ohta K, O’Byrne P, Pedersen SE, Pizzichini E, Sullivan SD, Wenzel SE, Zar HJ. Global strategy for asthma management and prevention: Gina executive summary. Eur Respir J. (2008); 31(1), 143-178. https://doi.org/10.1183/09031936.00138707

[6] Global initiative for asthma. Global srategy for asthma management and prevention. (2008). Retrieved April 15, 2009 from

http://www.ginasthma.org

[7] Wilson AJ, Gibson PG, Coughlan J. Long acting beta-agonists versus theophylline for maintenance treatment of asthma. Cochrane Database Syst Rev. (2003); (3): 1-26. https://doi.org/10.1002/14651858.CD001281

[8] Ukena D, Harnest U, Sakalauskas R, Magyar P, Vetter N, Steffen H, Leichtl S, Rathgeb F, Keller A, Steinijans VW. Comparison of addition of theophylline to inhaled steroid with doubling of the dose of inhaled steroid in asthma. Eur Respir J. (1997); 10(12): 2754-2760. https://doi.org/10.1183/09031936.97.10122754

[9] Lim S, Jatakanon A, Gordon D, Macdonald C, Chung KF, Barnes PJ. Comparison of high dose inhaled steroids, low dose inhaled steroids plus low dose theophylline, and low dose inhaled steroids alone in chronic asthma in general practice. Thorax. (2000); 55(10): 837-841. https://doi.org/10.1136/thorax.55.10.837

[10] Baumann TW. Some thoughts on the physiology of caffeine in coffee - and a glimpse of metabolite profiling. Braz J Plant Physiol. (2006); 18(1): 243-251. https://doi.org/10.1590/S1677-04202006000100017

[11] Scheindlin S. A new look at the xanthine alkaloids. Mol Interv. (2007); 7(5): 236-242. https://doi.org/10.1124/mi.7.5.1

[12] Constantinides PP. Lipid microemulsions for improving drug dissolution and oral absorption: physical and biopharmaceutical aspects. Pharm Res. (1995); 12: 1561-1572. https://doi.org/10.1023/a:1016268311867

[13] Liandong H, Yang J, Liu W, Li L. Preparation and evaluation of Ibuprofen-loaded microemulsion form improvement of oral bioavailability. Drug Deliv. (2011); 18(1): 90-5. https://doi.org/10.3109/10717544.2010.522613

[14] Baker RC, Florence AT, Tadros TF, Wood RM. Investigations into the formation and characterization of microemulsions. J Colloid Interface Sci. (1984); 100: 311-331. https://doi.org/10.1016/0021-9797(84)90438-7

[15] International Conference on Harmonization (ICH) Q2 (R1) Validation of analytical procedures: text and methodology. In: Brussels, Belgium: International Conference on Harmonisation. 1996.

[16] The United States Pharmacopeia: USP 29. Rockville, MD. (2006)

[17] Figueiredo KA, Neves JKO, Silva JAD, Freitas RMD, Carvalho ALM. Phenobarbital loaded microemulsion: development, kinetic release and quality control. Braz J Pharm Sci. (2016); 52(2): 251-264. https://doi.org/10.1590/S1984-82502016000200003

[18] Ozturk AA, Güven UM. Cefaclor monohydrate loaded microemulsion formulation for topical application: Characterization with new developed UPLC method and stability study. J Pharm Res. (2019); 23 (3): 426-440. https://doi.org/10.12991/jrp.2019.150

[19] Okur NU, Apaydin S, Yavasoglu NU, Yavasoglu, A, Karasulu HY. Evaluation of skin permeation and anti-inflammatory and analgesic effects of new naproxen microemulsion formulations. Int $J$ Pharm. (2011); 416(1): 136-144. https://doi.org/10.1016/j.ijpharm.2011.06.026 
[20] Piao HM, Balakrishnan P, Cho HJ, Kim H, Kim YS, Chung SJ, Shim CK, Kim DD. Preparation and evaluation of fexofenadine microemulsions for intranasal delivery. Int J Pharm. (2010); 395(12): 309-316. https://doi.org/10.1016/j.ijpharm.2010.05.041

[21] Shah RM, Malherbe F, Eldridge D, Palombo EA, Harding IH Physicochemical characterization of solid lipid nanoparticles (SLNs) prepared by a novel microemulsion technique. J Colloid Interface Sci. (2014); 428: 286-294.

https://doi.org/10.1016/j.jcis.2014.04.057

[22] Güven UM, Berkman MS, Yazan Y. Development and validation of UPLC method for the determination of olopatadine hydrochloride in polymeric nanoparticles. Acta Pharm Sci. (2019); 57 (1): 7-18. https://doi.org/10.23893/1307-2080.APS.05701

[23] Okur NU, Caglar ES, Yozgatli, V. Development and validation of an HPLC method for voriconazole active substance in bulk and its pharmaceutical formulation. Marmara Pharm J. (2016); 20(2): 79-85. https://doi.org/ 10.12991/mpj.20162076793

[24] Bhadra S, Das SC, Roy S, Arefeen S, Rouf ASS. Development and validation of RP-HPLC method for quantitative estimation of vinpocetine in pure and pharmaceutical dosage forms. Chromatogr Res Int. (2011); ID 801656. https://doi.org/10.4061/2011/801656

[25] Sintov AC, Brandys-Sitton R. Facilitated skin penetration of lidocaine: combination of a short-term iontophoresis and microemulsion formulation. Int J Pharm. (2006); 316(1-2): 58-67. https://doi.org/10.1016/j.ijpharm.2006.02.034
[26] Arevalo MI, Escribano E, Calpena A, Domenech J, Queralt J. Rapid skin anesthesia using a new topical amethocaine formulation: a preclinical study. Anesth Analg. (2004); 98(5): 1407-1412. https://doi.org/10.1213/01.ANE.0000107936.69436.5B

[27] Hathout RM, Woodman TJ, Mansour S, Mortada ND, Geneidi AS, Guy RH. Microemulsion formulations for the transdermal delivery of testosterone. Eur J Pharm Sci. (2010); 40(3): 188-196. https://doi.org/10.1016/j.ejps.2010.03.008

[28] Cao M, Ren L, Chen G. Formulation optimization and ex vivo and in vivo evaluation of celecoxib microemulsion-based gel for transdermal delivery. AAPS J. (2017); 18(6): 1960-1971. https://doi.org/10.1208/s12249-016-0667-z

[29] Gurpreet K, Singh SK. Review of nanoemulsion formulation and characterization techniques. Indian J Pharm Sci. (2018); 80(5): 781789. https://doi.org/10.4172/pharmaceutical-sciences.1000422

[30] Momoh MA, Franklin KC, Agbo CP, Ugwu CE, Adedokun MO, Anthony OC, Chidozie OM, Okorie AN. Microemulsion-based approach for oral delivery of insulin: formulation design and characterization. Heliyon. (2020); 6(3): e03650. https://doi.org/10.1016/j.heliyon.2020.e03650

[31] Ustundag Okur N, Caglar ES, Arpa MD, Karasulu HY. Preparation and evaluation of novel microemulsion-based hydrogels for dermal delivery of benzocaine. Pharm Dev Technol. (2017); 22(4): 500-510. https://doi.org/10.3109/10837450.2015.1131716

[32] Li X, Nie SF, Kong J, Li N, Ju CY, Pan W. A controlled-release ocular delivery system for ibuprofen based on nanostructured lipid carriers. Int J Pharm. (2008); 363(1-2): 177-182. https://doi.org/10.1016/j.ijpharm.2008.07.017 